DESY HERA 05-01

April 2005

Beam Losses and Machine Protection

K. Wittenburg

Deutsches Elektronen-Synchrotron DESY, Hamburg 


\title{
Beam Losses and Machine Protection
}

\author{
Kay Wittenburg \\ DESY, Hamburg
}

Talk given on $33^{\text {rd }}$ ADVANCED BEAM DYNAMICS WORKSHOP on HIGH INTENSITY \& HIGH BRIGHTNESS HADRON BEAMS; Bensheim, Germany, 10-22.Oct. 2004

\begin{abstract}
Large hadron accelerators (existing and planned) can have enough stored beam energy to have dangerous effects on the accelerator and its environment in case of uncontrolled beam losses. This report will give examples of different kinds of beam losses together with the response of the beam loss monitors (BLMs), the machine protection system (MPS) and adjacent implications. Expected and unexpected events from HERA and their impacts on the MPS will be presented and analyzed.
\end{abstract}

\section{INTRODUCTION}

Accidental beam losses of high energy, high brilliance or high intense beams can cause serious problems in accelerators including vacuum leaks, melting of material, activation, quenches of superconductors, etc. One of the most sensitive parts of the HERA proton ring (HERAp) to beam losses are the 648 superconducting main dipoles and quadrupoles around the $6.3 \mathrm{~km}$ long ring. This is also true for the LHC, with the additional complication that an uncontrolled loss of a substantial part of the LHC beam is able to destroy prominent parts of the beam pipe or of the collimator system. A beam loss monitor (BLM) system should measure all losses and should prevent dangerous beam loss rates in the machine. However, it can only take action, if already losses happened and therefore it stands in the very last position in a machine protection system. In the following we will discuss the experience of accidental beam losses at HERAp with the emphasis on beam loss induced quenches of superconducting magnets.

\section{The HERA MPS and BLM System}

The HERAp BLM system is described in detail in Ref [1] and the HERAp MPS in Ref. [2]. Only a short summary will be given here: The BLM consists of two PIN diodes, mounted faceto-face and read out in coincidence to suppress signals from synchrotron radiation of the adjacent electron accelerator. Charged particles, created by beam losses, are counted when crossing the two diodes. The counts are integrated over a time-period of $5.2 \mathrm{~ms}$ (about 250 turns). This count rate is proportional to the rate of beam particle losses at that location. The monitors are located on top of each superconducting quadrupole and on a large number of normal conducting quadrupoles in the straight sections of HERAp, especially at locations with a large $\beta$-function. Additional monitors are installed on the collimators and inside the experiments $\mathrm{H} 1$ and ZEUS. The dark count rate of the monitors is typically less than $0.1 \mathrm{~Hz}$, while the maximum count rate is the bunch crossing frequency of $10.4 \mathrm{MHz}$, which gives a dynamic range of more than $10^{8}$. A beam energy dependent upper threshold is active for each individual monitor. If the count rate reaches the threshold, an alarm is generated for the whole 
integration period. The alarm is hard wired to an alarm loop module close to the BLM readout electronics. This alarm module is hard wired connected to the central alarm loop module, which counts the number of BLM alarms. A threshold in the central alarm module defines the allowed number of BLM alarms. The threshold is set to 30 at injection and to 5 for all other machine conditions. The beam is dumped within one turn as soon as there are equal or more alarms than the threshold at the same time and the alarm signal was sent to the dump-electronics. A freeze signal stops the data acquisition of all BLMs (and BPMs) about $50 \mathrm{~ms}$ after an alarm. The loss rate of 128 time bins (each $5.2 \mathrm{~ms}, 665.6 \mathrm{~ms}$ total, "short mode") are stored in the alarm archive and can be viewed and annotated by the BLM-GUI of the HERA control system. Additionally, the mean loss rates of 128 short mode samples (85.2 s total, "long mode") are stored and can also be viewed. An example of a beam abort due to a BLM alarm is given in Fig. 1.

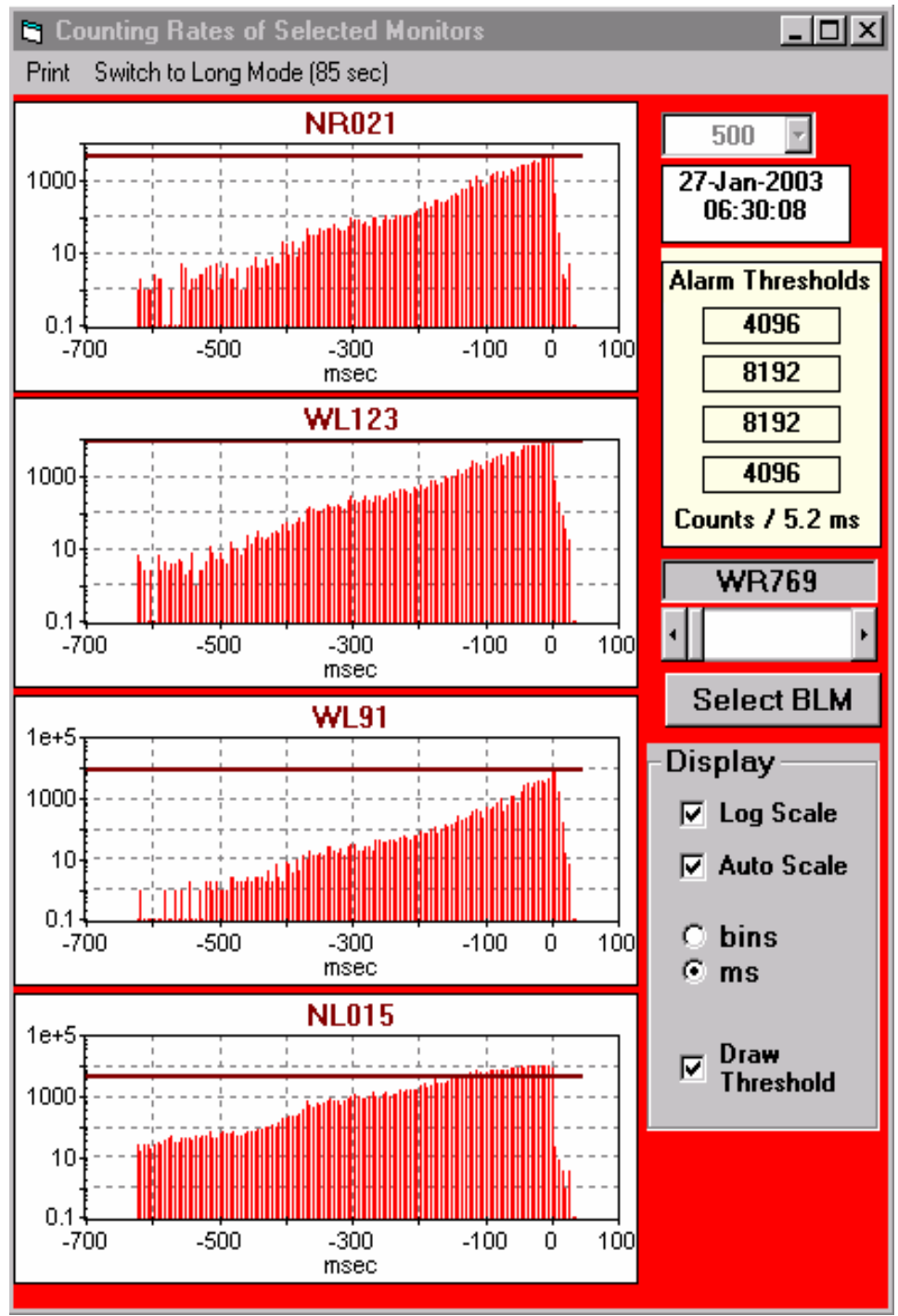

FIGURE 1. The increasing loss rate of 4 out of 302 BLMs in HERAp. The increasing vertical lines correspond to the loss rate within $5.2 \mathrm{~ms}$ (short mode). The big horizontal lines represent the individual thresholds. As soon as the last monitor reached the threshold (here WL91), the beam was dumped. The decreasing rates after the dump (at "0") are due to activation of the material. 


\section{Statistics}

HERA is operating since 1993. Major upgrades were done in 1998 and 2001 to increase the luminosity. The BLMs were installed in 1993 and the system operates since this time without significant changes. A few monitors were added to monitor magnets installed for the luminosity upgrade. The individual thresholds for the BLMs at the superconducting magnets were calculated by Monte Carlo simulations and were found to be reasonable. The settings at the warm magnets were also calculated and fine tuned (within a factor 2 or so) by experience. The very effective and reliable BLM archive system was used to analyze all beam loss induced beam aborts since 1994: Up to today (1.11.2004) we observed in HERAp 1218 beam loss induced events of which 199 led to a quench of magnets. Not in this analysis are the more than 1000 beam dumps due to hardware failures detected by the alarm loop (e.g. magnet power supplies trips), which led to clean beam dumps without additional problems. In the following the 199 beam loss induced quenches will be discussed and the reasons for the quenches in the presence of a BLM system will be analyzed.

\section{Events}

Fig. 2 gives an overview over the typical reasons for loss-induced quenches. One should note that a quench in HERAp is not a disaster. Typically the HERAp quench protection system detects the quench, fires the beam dump and organizes the quench procedure and recovery (Ref. 2). HERAp needs about 1-2 hours to recover from a quench (cryogenic) and can then continue with operation.

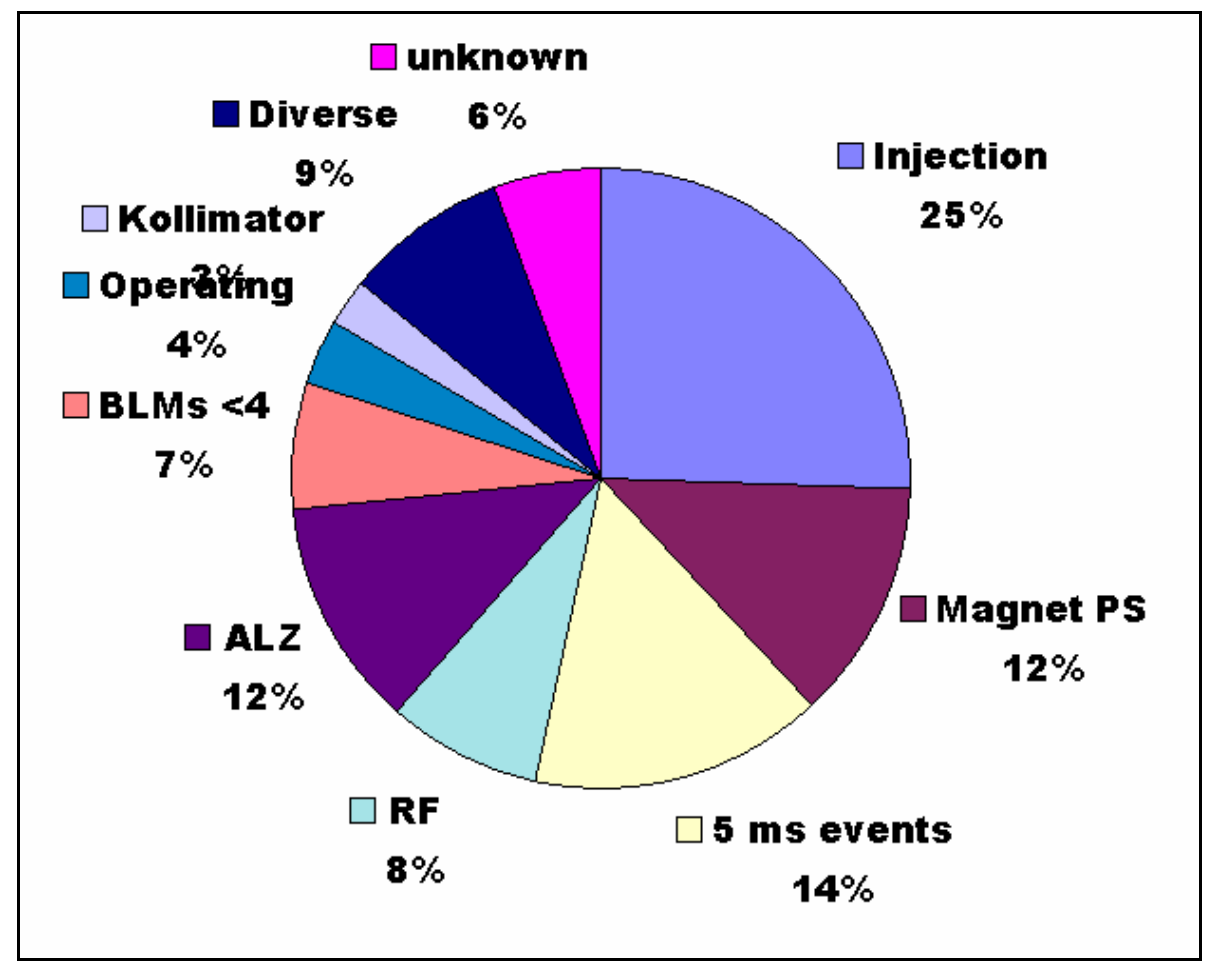

FIGURE 2. Percentage of reasons for beam loss induced quenches. Aberrations see text. 
Even in the beginning of the operation of HERA, beam losses were observed which occur very fast. The signature of these events in the BLM archive looked similar, with strong losses at many BLMs within the last $5.2 \mathrm{~ms}$ before the beam dump. The BLM system aborted the beam, but unfortunately the losses were high enough to quench magnets. Fig. 3 illustrates the typical BLM response. Because of their signature, the events were called "5 ms events", but the losses might occur faster than this. During the first years of operation, these events were not well understood.

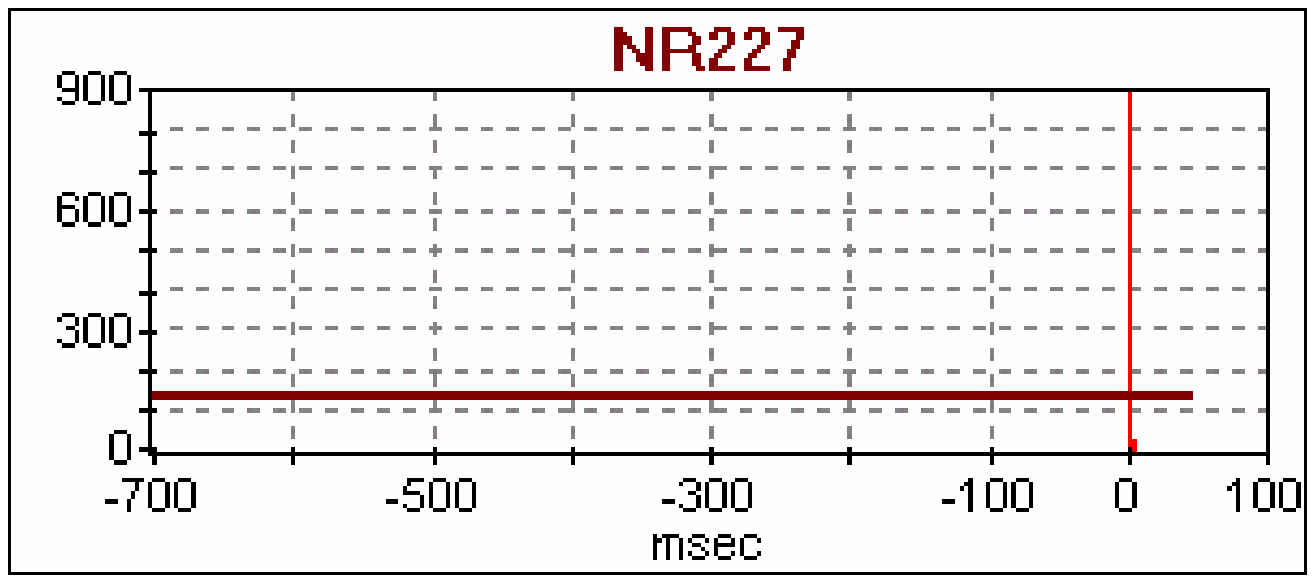

FIGURE 3. Typical response of a BLM induced by a "5 ms event". The horizontal line is the BLM threshold and the vertical line at "0" is the BLM count rate. No other counts within the last $665.6 \mathrm{~ms}$ were observed

The RF system was connected to the HERA alarm loop in 1998. This reduced the rate of $5 \mathrm{~ms}$ events and other beam loss induced beam aborts by roughly a factor two.

There was still a remaining event rate of about 0.5 "5 ms events" per week. Probably some of the quenches were induced by strong losses from the adjacent electron ring of HERA (HERAe).

However, the "5 ms event" rate became more frequent and more critical after the HERA Luminosity upgrade in 2003. The $\beta$-functions in the interaction region (IR) were reduced and more quadrupoles were installed, some very close to the IR. The number of power supplies for quadrupoles in the high $\beta$ region increased from 6 to 14. These new power supplies have had an about factor 3 smaller reliability than the old but well known power supplies from before the upgrade. Both, the higher number of power supplies and their smaller reliability led to a much smaller mean time between failures.

Typically, the beam has a high sensitivity to these low- $\beta$-insertion quadrupoles. It was calculated that a $1 \%$ change of the magnet current already leads to dramatic beam losses. Therefore a trip of such a power supply will lead to very fast beam losses $(<<5 \mathrm{~ms})$ even if the time constant $\tau$ of such a coil is in the order of some hundred milliseconds (see Fig. 4). To overcome this problem, different solutions were realized. Fast systems were developed to monitor power supplies and the beam current to abort the beam before dangerous losses occur: 1) The internal power supply alarm was made faster, 2) a new magnet current monitor was added to the alarm loop, 3) a hardware based fast beam current decay monitor was built and added to the alarm loop and 4) some delays in the alarm loop and in the beam dump trigger 
system were removed. All solutions were successfully tested in the beginning of 2004. Two additional solutions were discussed:

1) Reduction of the integration time of the BLMs. This was discarded in order not to increase the sensitivity to short "spikes" in the beam loss rate, sometimes observed by the experiments. Such a "spike" may cause a trip in the experimental environments, but should not abort the beam

2) Generation of fast alarms by large excursions in the BPMs. This was successfully tested in 2004, but the setting of an appropriate threshold over the whole machine-cycle needs more experiences.

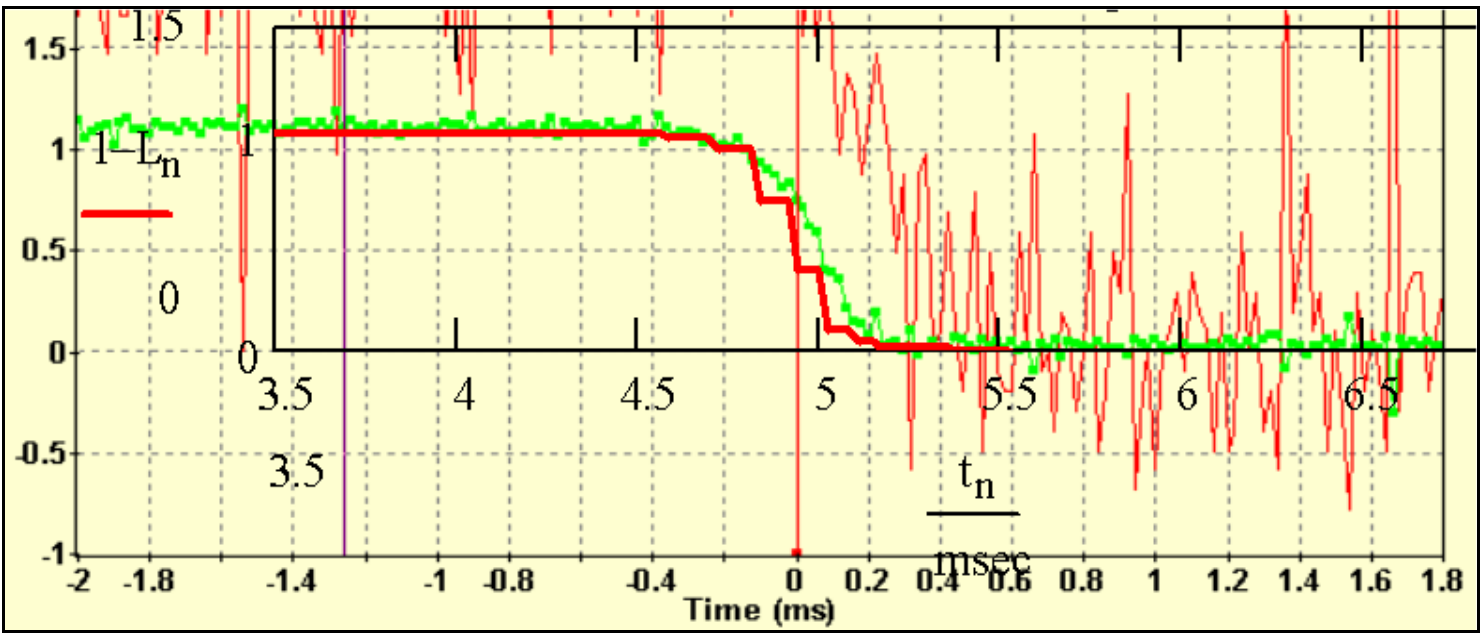

FIGURE 4. Measured and calculated beam loss after a trip of a GA-quadrupole. Green (longer) line: Fast beam current monitor, red (shorter) line: simulations. The vertical line at 0 indicates the alarm from the alarm loop about $4.9 \mathrm{~ms}$ after the trip of the GA magnet (with $\tau=524 \mathrm{~ms}$ ). From Ref. 3

\section{Injection}

Failures in the injection or ejection (dump) elements of HERA are expected to create very fast beam losses, for example due to misfired or not fired kickers. This type of failure happened about 2-3 times during the studied interval, while the main source of errors was an injection of the beam into a not sufficiently prepared machine. Typically an operator initiated the injection while HERAp was not at the right energy or other magnet settings were inadequate. Therefore the whole injected beam was lost into a few magnets within the first "turn". In principle these events should count to the "operating" topic, but as it is a significant rate of a special problem, it is counted as an individual topic. These events happened frequently in the very early years of HERA while in the last years it nearly vanished due to a well-trained crew. Nevertheless, this issue will be resolved soon by disabling the injection in case of non-suitable machine conditions.

\section{Alarm Loop (ALZ)}

The connection between the BLMs and the Alarm Loop has worked very reliable over the years. Even the huge amount of work close to the alarm loop during the luminosity upgrade did not reduce the reliability. However, since a lot of work may cause hardware problems, this 
happened also to the alarm loop connection: For the installation of a few new BLMs the cabling of some alarm-modules was renewed. Unfortunately two cables were exchanged at these modules, with the effect that the number of alarms were not added but subtracted. About 20 BLMs were affected, so that the threshold of 5 alarms was not valid as soon as these BLMs showed an alarm. Some quenches more occurred before one understood this scenario and repaired it. Meanwhile a procedure to check all BLM alarms has been established to avoid such problems.

As explained above, the alarm loop allows a certain number of BLM alarms before an emergency beam dump. This threshold is set by software via a fieldbus command. An improper design of the central alarm module led to some misinterpretations of fieldbus commands by the module, resulting in a larger threshold number. This led to some quenches because a much larger number of BLMs had to give an alarm before the beam was aborted.

A improved design of the central alarm loop module as well as some enhanced softwarecontrolling should avoid such events in the future.

\section{BLMs}

The calculated threshold for acceptable beam loss rates has turned out to be very well adjusted. The magnets can accept long losses just below the threshold without quenching, while losses just above the threshold have led to quenches (see Fig. 5).

The threshold settings in the warm part of HERAp were somewhat higher than in the superconducting part. Since the aperture limits are in the warm sections, one can expect higher losses and therefore higher values were chosen. While the loss rate threshold is chosen right, the threshold of how many BLM alarms will abort the beam is still in discussion. The rate of loss induced quenches due to a smaller number of BLM alarms $(<5)$ increased, especially after the luminosity upgrade. It turns out, that some losses are not obviously distributed over a larger area but localized at a few magnets. Therefore a threshold of 5 might be too high.

Despite of losses due to equipment failures, there are also losses due to other faulty machine conditions. So led a faulty reading of a Beam Position Monitor (BPM) to a local bump, which causes local losses at one BLM only. High and long term losses caused quenches at that location. An interesting event happened at a location just in the middle of the arc. There exist a special quadrupol without a BPM due to an additional cold box for Helium cooling. Since BLMs where ordered to install at all BPM locations, this quadrupole did not have a BPM as well as no BLM. It had cost some quenches to detect a local bump in this area (by analyzing the magnet settings), which caused the loss induced quenches of this magnet.

Two out of four loss induced quenches in 2004 happened in the superconducting magnets close to the IR. The thresholds of the BLMs at these magnets are set to relative high values and should be reduced by some factors in the near future. 

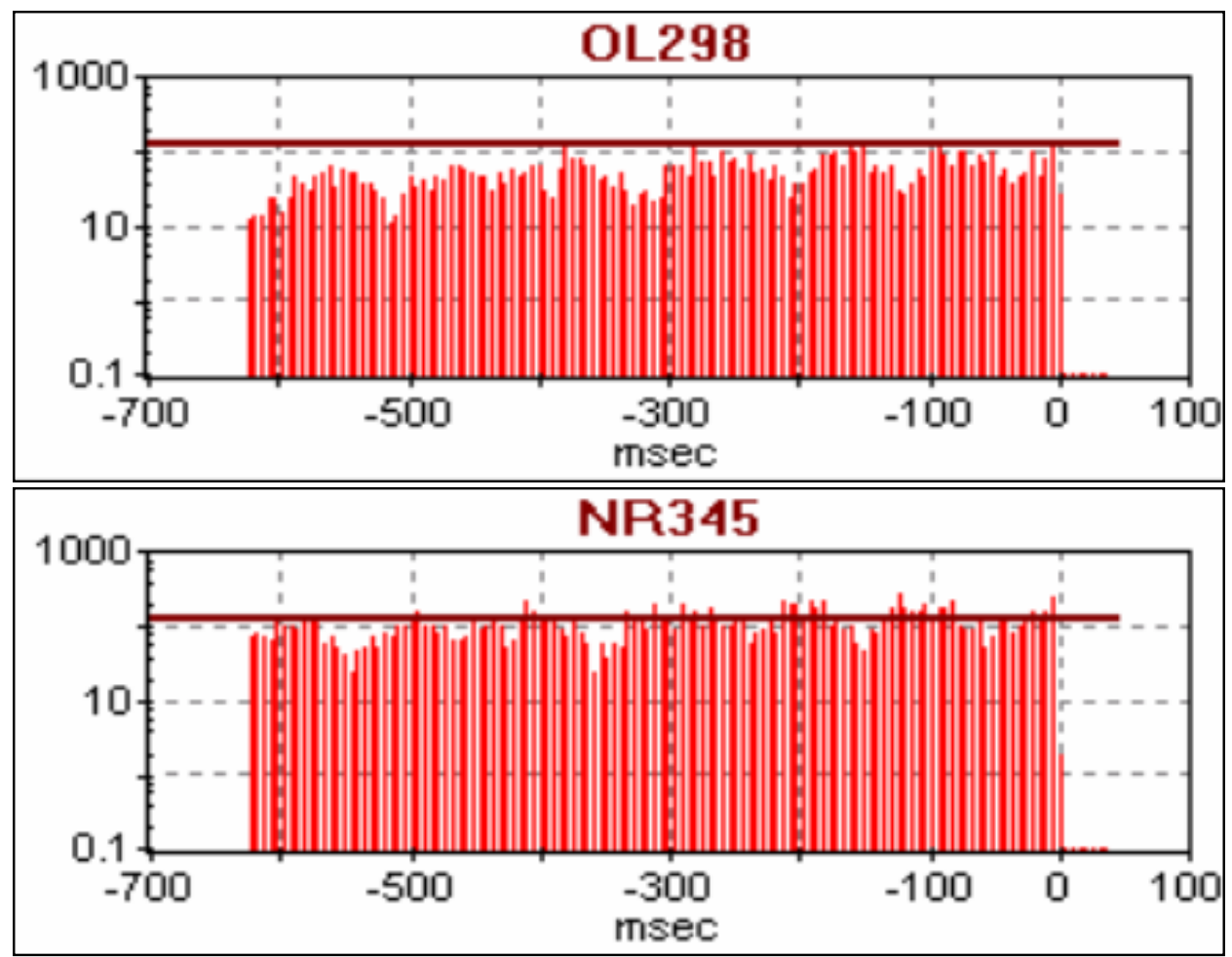

FIGURE 5: Loss rates and thresholds at two different BLMs without quench (upper) and with quench (lower) of the adjacent magnet for the same event. The acceptable loss rate for shorter losses is about a factor 5-10 higher than the threshold.

\section{Collimators}

The collimators of HERAp are designed to shield the experiments from extensive beam losses, but not necessarily the superconducting magnets. The closed collimators are the aperture limits of HERAp but scattered and shower particles can reach the following superconducting magnets and can cause a quench. One of the collimators is located just in front of the superconducting section. Therefore only a few BLMs are affected when having a high loss rate at that collimator. There are typically two BLMs on each collimator to double the number of alarms in such a case. However, the thresholds of these BLMs were increased over the years to avoid beam dumps due to high losses at the collimators. This caused no problem at normal operation and at "normal" loss conditions. But errors in the steering electronics of the collimators had caused some movements of the jaws much closer to the beam than expected. The resulting beam losses quenched the subsequent magnets.

It is foreseen to improve the reliability of the electronics of the collimators in the near future. Also the BLM thresholds at the critical collimator should be set somewhat lower.

\section{Operating, Miscellaneous and Unknown}

A number of quenches happened as a result of errors in operating the machine. But not only the crew in the main control room can have serious influences on the machine (machine studies, 
wrong files, ramp speed, RF settings, ...) but also some local controls: Losses and quenches have happened due to switching of power supplies during a running machine, installing or removing of test-equipment without communication and even drilling a hole into cables during operation. Also the experiments had caused events by driving equipment from or to the beam or by switching particular magnets.

Since a well-suited archive and logbook did not exist in the early years of HERAp, some quenches in that period were not documented and couldn't be classified.

As a consequence, a better education of the shift crew as well as the control crew of the experiments but also of the technical crews should be reached.

\section{SUMMARY}

Exact 199 accidental and uncontrolled beam losses happened in HERA and had led to beam loss induced quenches over a period of the past 10 years up to today (Sept. 2004). These events were analyzed and discussed with the emphasis on the reactions of the BLM system. More than $50 \%$ of the events were induced by very fast beam losses, which were faster than the designed reaction time of the BLM system $(5.2 \mathrm{~ms})$. Reasons for these events were injection errors (mainly operating) and failures of RF and special quadrupole power supplies. Hardware errors in the MPS of HERA caused $12 \%$ of beam loss induced quenches. The (safety-) concept of more than 4 coincident BLM alarms caused $7 \%$ of the quenches; somewhat faulty machine conditions had induce losses in one ore two magnets only. Missteered collimators, machine studies, operation errors, switching of equipment during running the machine, etc, generated other events but altogether these were of minor frequency.

A series of hardware improvements were done in HERAp, especially to analyze and to avoid the "5 ms events". Transient recorders already log signals from the RF, quenches, beam current, etc. Additional diag-nostic was installed to generate very fast alarms. But faster diagnostic is still required. Examples are: The BPM system already archives the last 1000 turns, but its alarmcapability is not adequate; fast logging and archiving of tune, chromaticity, longitudinal motions, emittance, etc. will support the analyses of events and may serve as additional fast alarm sources.

\section{ACKNOWLEDGMENTS}

Many thanks to Matthias Werner for his enthusiastic and rapid help in developing the fast beam current and magnet current protection systems, as well as for very valuable discussions and hints for increasing the speed of HERA's alarms. Also many thanks to M. Staack and to the quench protection crew for realizing the increased speed of the MPS. Greatly acknowledged are M. Lomperski's and F. Willeke's useful comments to the manuscript. P. Duval and M. Lomperski did an excellent job for the readout, archiving and displaying of the BLM data in the control room. 


\section{REFERENCES}

1. Kay Wittenburg, "The PIN-Diode Beam Loss Monitor System at HERA". in AIP Conference Proceedings Volume 546, pp. 3-22, 9th Beam Instrumentation Workshop, Boston, MA USA, May 8-11, 2000 and DESY-HERA-00-03 (Jun 2000).

2. R.Bacher, J. Bengtsson, G. Bialek,P. Duval, K.H.Mess, V.Nedic, M.Staack, L. Steffen, H.G. Wu; The HERA Quench Protection System: A Status Report, Sitges 1996, EPAC 96* pp 2264-2266 (1996). and DESY-M-96-13A, Jul 1996.

3. F. Willeke; DESY, private communication 